\title{
New evaluation of trunk movement and balance during walking in COPD patients by a triaxial accelerometer
}

This article was published in the following Dove Press journal: International Journal of COPD

\author{
Yoshino Terui' \\ Masahiro Iwakura ${ }^{1,2}$ \\ Eriko Suto ${ }^{3}$ \\ Atsuyoshi Kawagoshi ${ }^{2}$ \\ Keiyu Sugawara ${ }^{2}$ \\ Hitomi Takahashi ${ }^{2}$ \\ Kouichi Hasegawa ${ }^{3}$ \\ Sachiko Uemura' \\ Masahiro Satake' \\ Takanobu Shioya ${ }^{1,4}$ \\ 'Department of Physical Therapy, Akita \\ University Graduate School of Health \\ Sciences, Akita, Japan; ${ }^{2}$ Department \\ of Rehabilitation Medicine, Akita City \\ Hospital, Akita, Japan; ${ }^{3}$ Department \\ of Rehabilitation, Akita Prefectural \\ Center for Rehabilitation and \\ Psychiatric Medicine, Daisen, Japan; \\ ${ }^{4}$ Geriatric Health Services Facility \\ Nikonikoen, Akita, Japan
}

Correspondence: Yoshino Terui Department of Physical Therapy, Akita University Graduate School of Health Sciences, I-I-I Hondo, Akita 010-8543, Japan

Tel +8I I8 8846504 ext 6527

Fax +8I I8 8846500

Email terui@hs.akita-u.ac.jp
Background: Individuals with COPD may experience ambulatory difficulty due to both effort intolerance arising from respiratory dysfunction and impaired balance control during walking. However, the trunk movement during walking has not been evaluated or adjusted for patients with COPD. The Lissajous index (LI) visually and numerically evaluates the left-right symmetry of the trunk movement during walking and is useful in clinical practice. In COPD patients, the LI is used as an indicator of the left-right symmetry of the trunk during walking. Here, we used the LI to evaluate the symmetry of COPD patients based on bilateral differences in mediolateral and vertical accelerations, and we investigated the correlation between the patients' symmetry evaluation results and their physical function.

Patients and methods: Sixteen stable COPD patients (all males; age 71.3 \pm 9.2 years) and 26 healthy control subjects ( 15 males; age $68.2 \pm 6.9$ years) participated in this study. They performed the 10-minute walk test at a comfortable gait speed wearing a triaxial accelerometer, and we measured their trunk acceleration for the evaluation of symmetry. Motor functions were also evaluated in the patients with COPD.

Results: The average mediolateral bilateral difference and LI values of the COPD patients were significantly larger than those of the healthy subjects. The COPD patients' LI values were significantly correlated with their static balance.

Conclusion: The LI measured using a triaxial accelerometer during walking is useful in balance assessments of patients with COPD.

Keywords: chronic obstructive pulmonary disease, gait, accelerometer, Lissajou index, balance

\section{Introduction}

Individuals with COPD may have an ambulatory disability due to both effort intolerance arising from respiratory dysfunction and impaired gait parameters. The impairment of gait parameters includes the decreasing step length, cadence, and gait speed. ${ }^{1,2}$ Gait parameter deficits in daily living increase the risk of falling. ${ }^{1}$ Many studies have recorded the gait in patients with COPD using a three-dimensional (3D) motion capture system and a pressure-sensitive mat. ${ }^{3}$ A pressure-sensitive mat and 3D motion capture system can only assess a limited number of consecutive strides due to space and equipment constraints. ${ }^{4,5}$ In addition, $3 \mathrm{D}$ motion capture system is very expensive. ${ }^{6}$ In contrast, triaxial accelerometers are small and useful to measure gaits in various environments. ${ }^{7}$ Recent studies have reported that gait analysis using triaxial accelerometers has many advantages and reliability of measurement. ${ }^{8-11}$ Several gait parameters associated with acceleration have been developed, eg, the Lissajous index (LI) ${ }^{7}$ for 
evaluating gait symmetry of the trunk movement, the root mean square ${ }^{11,12}$ representing the average of trunk acceleration amplitudes, and the harmonic ratio ${ }^{13}$ indicating the smoothness of a gait.

In our previous study, the LI visually and numerically evaluated the left-right symmetry of a Lissajous figure (LF); this LF is made from trunk acceleration data. ${ }^{14}$ Further, it was clear that the LI during gait was effective at evaluating gait balance in patients with stroke. ${ }^{14}$ The gait parameter has been found useful for the patients with COPD, but the trunk movement during walking has not been evaluated or adjusted for patients with COPD. It was considered that the patients with COPD have the abnormal trunk movement during walking by impaired gait parameters.

The purpose of this study was to evaluate the trunk movement using the LF and the LI, to compare the LI with the uniaxial acceleration, and to examine the relation between LI and body function in patients with COPD.

\section{Patients and methods}

\section{Subjects}

Sixteen stable elderly COPD patients (all male; aged 71.3 \pm 9.2 years) who had undergone pulmonary rehabilitation at Akita City Hospital were enrolled in this study. Twenty-six healthy control subjects (15 males; aged 68.2 \pm 6.9 years) who had worked at the Akita Prefectural Center for Rehabilitation and Psychiatric Medicine were also enrolled. All subjects provided written informed consent.

The following patients were excluded: 1) patients who were not able to walk by themselves without canes, 2) those with an illness or injury other than COPD that impaired their behavior, and 3) those who did not understand the purpose of the experiment. The characteristics of the patients and healthy controls are summarized in Table 1.

\section{Study design}

For the measurement of trunk acceleration during walking, each subject wore the triaxial accelerometer described below and underwent a 10-minute gait test at a comfortable gait speed. The subject's symmetry during his walking was calculated based on his trunk acceleration. The patients with COPD also underwent an evaluation of their motor function. This study was reviewed and approved by the Ethics Committees of Akita University Graduate School of Medicine (2015; approval no 1319) and carried out in conformity with Declaration of Helsinki.
Table I Characteristics of the COPD patients and healthy controls

\begin{tabular}{|c|c|c|c|}
\hline Characteristic & COPD $(n=16)$ & Control $(n=26)$ & $P$-value \\
\hline Age, years & $71.3(9.2)$ & $68.2(6.9)$ & 0.263 \\
\hline Males, $\mathrm{n}$ & 16 & 15 & - \\
\hline Height, m & $165.0(5.1)$ & $160.6(9.6)$ & 0.059 \\
\hline Body weight, kg & $59.3(6.4)$ & $57.8(10.9)$ & 0.626 \\
\hline BMI, $\mathrm{kg} / \mathrm{m}^{2}$ & $21.8(2.9)$ & $22.3(2.5)$ & 0.620 \\
\hline GOLD, I/II/III/IV & $3 / 7 / 6 / 0$ & - & - \\
\hline $\mathrm{mMRC}, 0 / \mathrm{I} / 2 / 3 / 4$ & $0 / 7 / 5 / 4 / 0$ & - & - \\
\hline$\% F V C, \%$ predicted & $82.2(22.8)$ & - & - \\
\hline$\% \mathrm{FEV}_{1}, \%$ predicted & $58.4(20.1)$ & - & - \\
\hline $\mathrm{FEV}_{1} / \mathrm{FVC}, \%$ & $51.0(15.7)$ & - & - \\
\hline SPPB total, point & $11.5(3)$ & - & - \\
\hline OLST, seconds & $\mathrm{I} .0(0.2)$ & - & - \\
\hline WBI, \% & $0.7(0.2)$ & - & - \\
\hline 6MWD, minutes & $437.6(161.2)$ & - & - \\
\hline
\end{tabular}

Note: Data are represented as mean (SD) or median (interquartile range).

Abbreviations: 6MWD, the 6-minute walk distance; BMI, body mass index; GOLD, Global Initiative for Chronic Obstructive Lung Disease; mMRC, Modified Medical Research Council scale; OLST, one-leg standing test; SPPB, short physical performance battery; WBI, the muscle strength of leg with the weight bearing index of quadriceps.

\section{Accelerometer}

The small (20 g) wireless triaxial accelerometer (MG-M1110; LSI Medience, Tokyo, Japan $)^{9,10}$ is $75 \mathrm{~mm}$ long, $50 \mathrm{~mm}$ high, and $20 \mathrm{~mm}$ wide (Figure 1). The sampling rate was $100 \mathrm{~Hz}$. The accelerometer was used to measure the vertical (VT), anteroposterior (AP), and mediolateral (ML) acceleration of the subject's trunk during his gait. Each aspect of acceleration is indicated by graphs produced by the accelerometer's

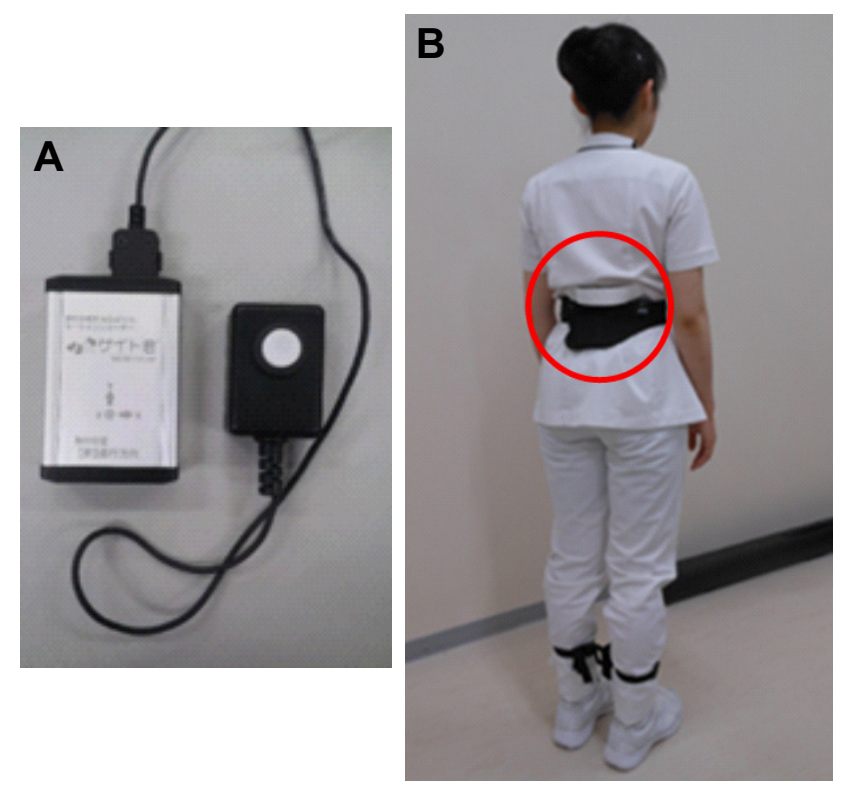

Figure I A triaxial accelerometer (MG-MIII0; LSI Medience, Tokyo, Japan). Notes: (A) A triaxial accelerometer. (B) A person wearing the device. 
analysis software for a personal computer (MG-M1110-PC ${ }^{\mathrm{TM}}$; LSI Medience). ${ }^{9,10}$ This software calculated the steps, gait speed, and gait coefficient of variation and exported the values of each aspect of acceleration into Microsoft's Excel program.

\section{Accelerometer measurements}

The accelerometer was fixed to a belt at the level of the subject's L3 spinous process (Figure 1). All subjects performed the 10 -minute walk test twice for the recording of definitive data on trunk acceleration. A 10-m-long walkway with a 1-m spare walkway area at both the start and the end was constructed. The subject was instructed to walk straight at a self-selected comfortable speed toward a target line on the floor, without the use of a cane. The acceleration measurements were performed twice by the same tester. The acceleration data of second walk test was used for analysis. All acceleration data from the 10-m walk except for the first and final steps were used.

\section{Symmetry evaluation (ML or VT acceleration)}

The MG-M1110 displays the ML acceleration waveforms as " $x$ " and the VT acceleration waveforms as " $y$ ". A positive $x$-value indicates acceleration to the left, and a negative $x$-value indicates acceleration to the right. Thus, for our comparison of the amount of acceleration occurring on the left and right, we calculated the maximum difference in the absolute value for lateral acceleration ( $\Delta x$ lateral). The difference between the maximum value for VT acceleration when the right leg is in the stance phase and the maximum value for VT acceleration when the left leg is in the stance phase ( $\Delta y$ lateral) was also calculated.

\section{Symmetry evaluation (Lissajou index)}

We calculated the LI, ${ }^{7,14}$ which indicates the symmetry based on both ML and VT accelerations. For the calculation of an LI, an LF must be drawn. Using Excel, the LF was graphed on a scatter chart with the $y$-axis representing VT acceleration and the $x$-axis representing ML acceleration. Figure 2 shows the LF made from actual measured values of trunk acceleration. The quadrangle surrounded by the undulating line $(\mathrm{Rr})$ and the quadrangle surrounded by the undulating and dotted lines ( $\mathrm{Rl}$ ) were the calculated areas (Figure 2).

The following formulae were used to calculate the values of $\mathrm{Rr}$ and $\mathrm{Rl}$ :

The length of the rectangle: [the maximum of vertical acceleration -0.98$]\left(0.98 \mathrm{~m} / \mathrm{s}^{2}\right.$ is the acceleration of gravity).

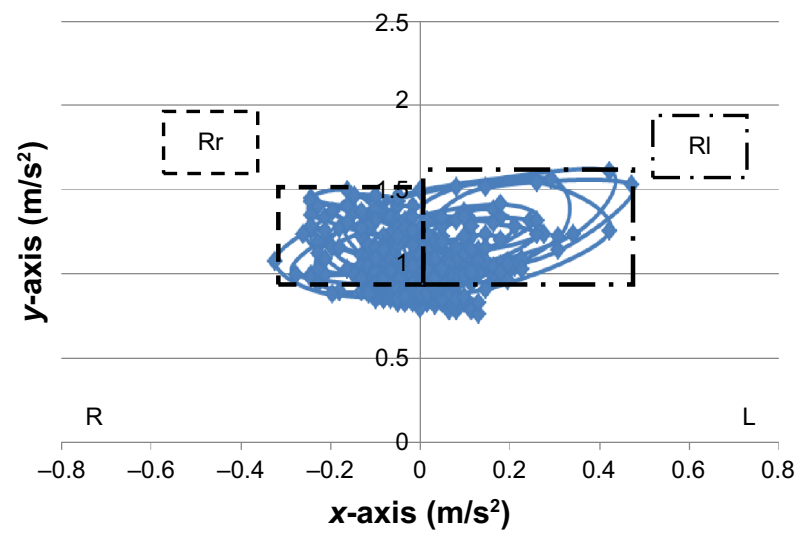

Figure 2 Lissajous figure.

Note: $y$-axis, vertical acceleration; $x$-axis, mediolateral acceleration. Abbreviations: L, left; $\mathrm{R}$, right; RI, rectangle area of left, the quadrangle surrounded by undulating and dotted line; $R r$, rectangle area of right, the quadrangle surrounded by undulating line.

The width of the rectangle: [the maximum absolute value of mediolateral acceleration]

The area of $\mathrm{Rr}$ and $\mathrm{Rl}$ : [the maximum of vertical acceleration -0.98$] \times[$ the maximum absolute value of mediolateral acceleration]

The formula for calculating the LI is as follows:

$$
L I=\left|\frac{2 \times(R r-R l)}{(R r+R l)} \times 100\right|
$$

A large LI value indicates asymmetry, and small LI values denote symmetry on the frontal plane.

\section{Motor function}

The severity level of the patients' COPD was evaluated with the Global Initiative for Chronic Obstructive Lung Disease (GOLD), ${ }^{15}$ and the patients' dyspnea was evaluated with the Modified Medical Research Council scale. ${ }^{16}$ For the pulmonary function, we measured the \%FVC $(\%$ forced volume capacity), $\% \mathrm{FEV}_{1}$, and $\mathrm{FEV}_{1} / \mathrm{FVC}$ with the use of a spirometer (Chestgraph Jr. HI-101, Chest M.I., Tokyo, Japan). ${ }^{17}$ The static balance was evaluated with the one-leg standing test (OLST), and the dynamic balance was evaluated with the short physical performance battery (SPPB) ${ }^{18}$ and the muscle strength of leg with the Weight Bearing Index of quadriceps. ${ }^{19}$ The 6-minute walk distance was measured as an indicator of functional exercise capacity. ${ }^{20}$ Motor function was evaluated only in the COPD patients. 


\section{Statistical analyses}

The LI, $\Delta x$ lateral, and $\Delta y$ lateral values in the COPD group and control group were compared with an unpaired $t$-test. The relationships of the $\Delta x$ lateral and LI values in the COPD group with all physical functions were investigated using Pearson correlation coefficient for analyses on quantitative variables and Spearman correlation in the case of ordinal variables. The statistical software SPSS Statistics 24 was used for the analyses. The level of significance was set at $5 \%$.

\section{Results}

Figure 3 shows the actual LF of the healthy subjects and the patients with COPD. From this figure, it is clear that the LFs of the patients with COPD were larger and more asymmetric than the LFs of the healthy subjects. Table 2 illustrates the LI, the $\Delta x$ lateral, and the $\Delta y$ lateral of the patients with COPD and the healthy subjects. An unpaired $t$-test revealed that both the average LI value and the $x$-bilateral difference of the COPD patients were significantly larger than those of the healthy subjects ( $P=0.02$ and $P=0.007$, respectively).

The results of the correlation analysis are given in Table 3 . The LI values of the patients with COPD were significantly correlated with their OLST results $(P=0.042)$.

\section{Discussion}

In this study, the trunk acceleration of patients with COPD and healthy subjects was measured during walking at a comfortable speed for 10 minutes by a triaxial accelerometer at the waist. To evaluate the trunk movement during walking, the $\Delta x$ lateral, $\Delta y$ lateral, and LI values were calculated based on the acceleration data. The $\Delta x$ lateral value was significantly higher in the COPD group compared with the

A

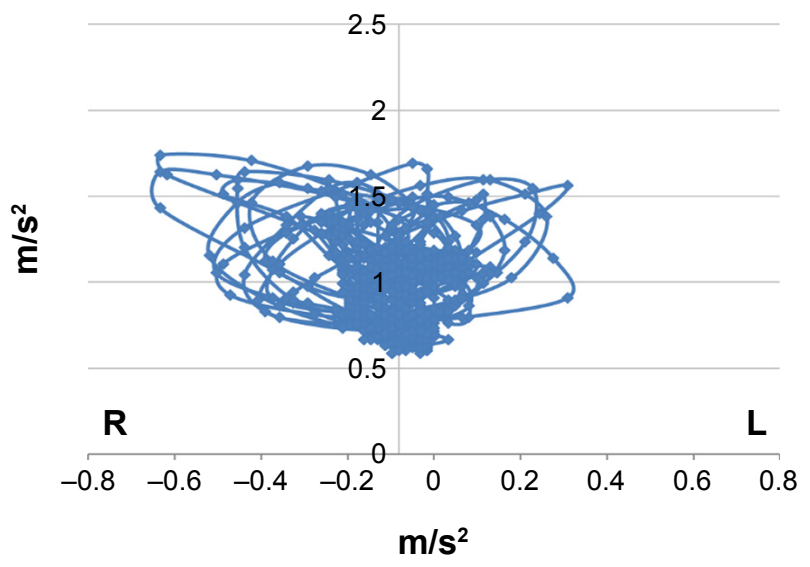

healthy controls. The $\Delta y$ lateral demonstrated no significant difference between the two groups. The LI of the COPD patients was significantly higher than that of the healthy controls, and the LI was shown to be related to the patients' standing balance ability.

The $\Delta x$ lateral value was significantly higher in the COPD group compared with the healthy controls. Lim et al ${ }^{21}$ reported that decreased movement in the gluteal muscles and the erector spinae muscle in elderly individuals leads to increases in trunk acceleration in the lateral direction. It has also been indicated that COPD often exhibits severe muscle wasting. ${ }^{22}$ It is thus possible that decreased muscle strength in the trunk and legs of patients with COPD has an effect on the lateral asymmetry of both their lateral acceleration and trunk acceleration.

We observed that the LI values of the COPD patients were significantly higher than those of the healthy subjects. The LI values of the COPD group were also correlated with their results on the OLST, which assesses standing balance. In a previous study, the standing balance ability in COPD patients was lower than that of healthy subjects. ${ }^{23}$ Our present group of COPD patients was composed of patients who did not have conditions that affected one side of their bodies, such as hemiplegia or orthopedic disorders. Our findings indicate that decreased balance ability in patients with COPD is related to their increased lateral asymmetry during walking.

Among the evaluations of trunk movement in the present study, the LI of COPD patients was the only measurement that was not only significantly larger than the LI of healthy individuals but also related to standing balance. In recent years, there has been increasing research focusing on the decrease in COPD patients' balance ability. ${ }^{24,25}$

B

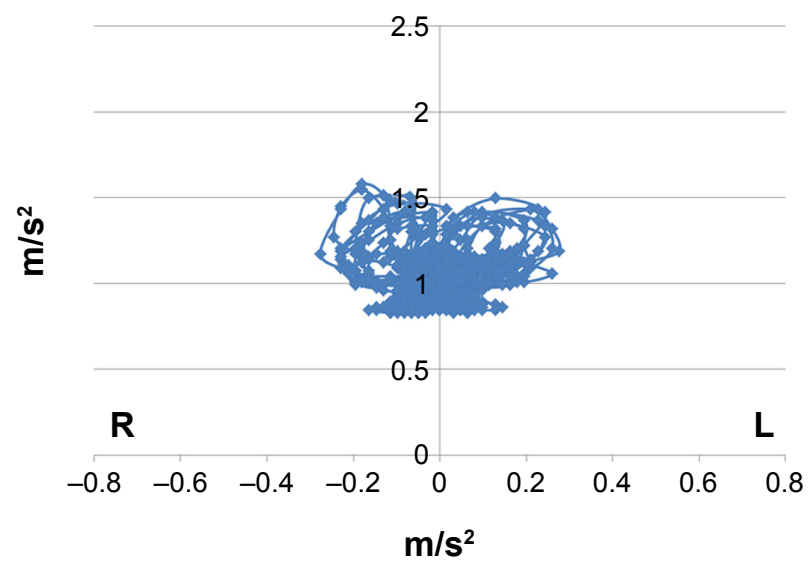

Figure 3 Actual Lissajous figures.

Notes: (A) The Lissajous figure of a COPD patient. (B) The Lissajous figure of a healthy individual.

Abbreviations: L, left; R, right. 
Table 2 Symmetry evaluation of COPD patients and healthy control

\begin{tabular}{l|l|l|l}
\hline $\begin{array}{l}\text { Outcome } \\
\text { measure }\end{array}$ & COPD & Control & P-value \\
\hline $\mathrm{LI} \%$ & $34.2(19.2)^{*}$ & $15 . \mathrm{I}(\mathrm{II} . \mathrm{I})$ & 0.002 \\
$\Delta x$ lateral, g & $0.22(0.15)^{*}$ & $0.10(0.12)$ & 0.007 \\
$\Delta y$ lateral, g & $0.15(0.1 \mathrm{I})$ & $0.12(0.10)$ & $0.39 \mathrm{I}$ \\
\hline
\end{tabular}

Notes: Data are represented as mean (SD). *Differences are significant. Abbreviation: LI, Lissajous index.

Bernabeu-Mora et al reported the correlation between impairment of balance ability and physical function using each test of the SPPB, which assesses dynamic balance. ${ }^{26}$ Several reports indicated that decreases in the level of physical activity, which affect the prognosis of patients with COPD, can cause reduced balance ability. ${ }^{27-29}$ Compared with healthy individuals, COPD patients take a longer time to correct losses of balance while walking, and they require more trunk muscle activity. ${ }^{30}$ If patients with COPD fall and injure themselves, they may experience decreased physical functions, and their resulting reluctance to walk may lead to decreased levels of physical activity. ${ }^{31,32}$

Based on these findings, it is extremely important to use an approach for COPD patients that assesses their balance while walking. Based on the presented findings, the LI measured using a triaxial accelerometer is useful in balance assessments.

This study had three limitations. First, the number of subjects analyzed was small. For the precise determination

Table 3 Correlation coefficient between symmetry evaluation and motor function in patients with COPD

\begin{tabular}{l|l|l}
\hline Motor functions & LI & $\Delta$ x lateral \\
\hline Age & -0.328 & 0.123 \\
Height & 0.225 & -0.188 \\
Body weight & 0.333 & 0.060 \\
BMI & 0.143 & 0.124 \\
GOLD & -0.319 & -0.008 \\
mMRC & -0.425 & 0.288 \\
\%FVC & 0.227 & 0.095 \\
\%FEV, & 0.254 & 0.037 \\
FEV,FVC & 0.154 & -0.155 \\
SPPB total & -0.019 & -0.236 \\
OLST & $-0.530 *$ & -0.220 \\
WBI & 0.195 & -0.365 \\
6MWD & 0.340 & -0.197 \\
\hline
\end{tabular}

Notes: The values are Pearson's product-moment correlation coefficient or Spearman's rank correlation coefficient. $* P<0.05$.

Abbreviations: 6MWD, the 6-minute walk distance; BMI, body mass index; GOLD, Global Initiative for Chronic Obstructive Lung Disease; LI, Lissajous index; mMRC Modified Medical Research Council scale; OLST, one-leg standing test; SPPB, short physical performance battery; WBI, the muscle strength of leg with the weight bearing index of quadriceps. of the usefulness of balance assessments during walking in patients with COPD, further studies are required with greater numbers of subjects, including those with stage IV conditions. Second, the lateral differences in the legs' muscle strength were not evaluated in both the COPD and healthy groups. Third, we did not measure the leg or trunk muscle strength of any muscle other than the quadriceps. Trunk acceleration is affected by the muscles of the trunk, the gluteal region, and the legs. Further research that measures the muscle strength in the other regions is required.

\section{Conclusion}

The present study is the first research of the trunk movement during walking in the patients with COPD. The lateral symmetry of COPD patients during walking could be evaluated based on the LI obtained by a triaxial accelerometer. In addition, the LI values of the COPD patients were associated with their standing balance. The ambulatory disability of COPD patients is affected by not only their effort intolerance due to respiratory dysfunction but also their underlying balance control. If patients with COPD fall and injure themselves, they may experience decreased physical functions, and their resulting reluctance to walk may lead to decreased levels of physical activity. It is very important to use an approach that assesses balance while walking in patients with COPD. Our present findings demonstrate that the LI is a useful parameter for virtual and numerical evaluations of the walking balance of individuals with COPD.

\section{Disclosure}

The authors report no conflicts of interest in this work.

\section{References}

1. Annegarn J, Spruit MA, Savelberg HH, et al. Differences in walking pattern during 6-min walk test between patients with COPD and healthy subjects. PLoS One. 2012;7(5):e37329.

2. Karpman C, Benzo R. Gait speed as a measure of functional status in COPD patients. Int J Chron Obstruct Pulmon Dis. 2014;9(9):1315-1320.

3. Lahousse L, Verlinden VJ, van der Geest JN, et al. Gait patterns in COPD: the Rotterdam Study. Eur Respir J. 2015;46(1):88-95.

4. Yentes JM, Schmid KK, Blanke D, Romberger DJ, Rennard SI, Stergiou N. Gait mechanics in patients with chronic obstructive pulmonary disease. Respir Res. 2015;16:31.

5. Howell D, Osternig L, Chou LS. Monitoring recovery of gait balance control following concussion using an accelerometer. J Biomech. 2015; 48(12):3364-3368.

6. Henriksen M, Lund H, Moe-Nilssen R, Bliddal H, Danneskiod-Samsøe B. Test-retest reliability of trunk accelerometric gait analysis. Gait Posture. 2004;19(3):288-297.

7. Yamaguchi R, Hirata S, Doi T, Miura Y. The usefulness of a new gait symmetry parameter derived from Lissajous figures of tri-axial acceleration signals of the trunk. J Phys Ther Sci. 2012;24(5):405-408.

8. Osaka H, Shinkoda K, Watanabe S, et al. Association between trunk acceleration during walking and clinically assessed balance in patients with stroke. Neuro Rehabilitation. 2017;41(4):783-790. 
9. Yoneyama M, Mitoma H, Okuma Y. Accelerometry-based long-term monitoring of movement disorders: from diurnal gait behavior to nocturnal bed mobility. J Mech Med Biol. 2013;13(2):1350041.

10. Yoneyama M, Kurihara Y, Watanabe K, Mitoma H. Accelerometrybased gait analysis and its application to Parkinson's disease assessment- part 2: a new measure for quantifying walking behavior. IEEE Trans Neural Syst Rehabil Eng. 2013;21(6):999-1005.

11. Moe-Nilssen R. Test-retest reliability of trunk accelerometry during standing and walking. Arch Phys Med Rehabil. 1998;79(11):1377-1385.

12. Sekine M, Tamura T, Yoshida M, et al. A gait abnormality measure based on root mean square of trunk acceleration. J Neuroeng Rehabil. 2013;10:118.

13. Brach JS, McGurl D, Wert D, Vanswearingen JM, Perera S, Cham R, Studenski S. Validation of a measure of smoothness of walking. J Gerontol A Biol Sci Med Sci. 2011;66(1):136-141.

14. Terui Y, Suto E, Konno Y, et al. Evaluation of gait symmetry using a tri-axial accelerometer in stroke patients. Neuro Rehabilitation. 2018; 42(2):173-180.

15. Vestbo J, Hurd SS, Agustí AG, et al. Global strategy for the diagnosis, management, and prevention of chronic obstructive pulmonary disease: GOLD executive summary. Am J Respir Crit Care Med. 2013;187(4): 347-365.

16. Celli BR, MacNee W; ATS/ERS Task Force. Standards for the diagnosis and treatment of patients with COPD: a summary of the ATS/ERS position paper. Eur Respir J. 2004;23(6):932-946.

17. Standardization of spirometry - 1987 update. Statement of the American Thoracic Society. Am Rev Respir Dis. 1987;136(5):1285-1298.

18. Guralnik JM, Simonsick EM, Ferrucci L, et al. A short physical performance battery assessing lower extremity function: association with self-reported disability and prediction of mortality and nursing home admission. J Gerontol. 1994;49(2):M85-M94.

19. Sugawara K, Takahashi H, Kashiwagura T, et al. Effect of antiinflammatory supplementation with whey peptide and exercise therapy in patients with COPD. Respir Med. 2012;106(11):1526-1534.

20. ATS Committee on Proficiency Standards for Clinical Pulmonary Function Laboratories. ATS statement: guidelines for the six-minute walk test. Am J Respir Crit Care Med. 2002;166(1):111-117.

21. Lim YP, Lin YC, Pandy MG. Muscle function during gait is invariant to age when walking speed is controlled. Gait Posture. 2013;38(2): 253-259.
22. Skeletal muscle dysfunction in chronic obstructive pulmonary disease. A statement of the American Thoracic Society/European Respiratory Society. Am J Respir Crit Care Med. 1999;159(4 Pt 2):S1-S40.

23. Iwakura M, Okura K, Shibata K, Kawagoshi A, Sugawara K, Takahashi H, Shioya T. Relationship between balance and physical activity measured by an activity monitor in elderly COPD patients. Int J Chron Obstruct Pulmon Dis. 2016;11:1505-1514.

24. Roig M, Eng JJ, MacIntyre DL, Road JD, FitzGerald JM, Burns J, Reid WD. Falls in people with chronic obstructive pulmonary disease: an observational cohort study. Respir Med. 2011;105(3):461-469.

25. Porto EF, Castro AA, Schmidt VG, Rabelo HM, Kümpel C, Nascimento OA, Jardim JR. Postural control in chronic obstructive pulmonary disease: a systematic review. Int J Chron Obstruct Pulmon Dis. 2015;10:1233-1239.

26. Bernabeu-Mora R, Giménez-Giménez LM, Montilla-Herrador J, GarcíaGuillamón G, García-Vidal JA, Medina-Mirapeix F. Determinants of each domain of the Short Physical Performance Battery in COPD. Int J Chron Obstruct Pulmon Dis. 2017;12:2539-2544.

27. Beauchamp MK, Sibley KM, Lakhani B, Romano J, Mathur S, Goldstein RS, Brooks D. Impairments in systems underlying control of balance in COPD. Chest. 2012;141(6):1496-1503.

28. Voica AS, Oancea C, Tudorache E, Crisan AF, Fira-Mladinescu O, Tudorache V, Timar B. Chronic obstructive pulmonary disease phenotypes and balance impairment. Int J Chron Obstruct Pulmon Dis. 2016;11:919-925.

29. Kawagoshi A, Kiyokawa N, Sugawara K, Takahashi H, Sakata S, Satake M, Shioya T. Effects of low-intensity exercise and home-based pulmonary rehabilitation with pedometer feedback on physical activity in elderly patients with chronic obstructive pulmonary disease. Respir Med. 2015;109(3):364-371.

30. Smith MD, Chang AT, Hodges PW. Balance recovery is compromised and trunk muscle activity is increased in chronic obstructive pulmonary disease. Gait Posture. 2016;43:101-107.

31. Crişan AF, Oancea C, Timar B, Fira-Mladinescu O, Tudorache V. Balance impairment in patients with COPD. PLoS One. 2015;10(3): e0120573.

32. Tudorache E, Oancea C, Avram C, Fira-Mladinescu O, Petrescu L, Timar B. Balance impairment and systemic inflammation in chronic obstructive pulmonary disease. Int J Chron Obstruct Pulmon Dis. 2015; 10:1847-1852.
International Journal of COPD

\section{Publish your work in this journal}

The International Journal of COPD is an international, peer-reviewed journal of therapeutics and pharmacology focusing on concise rapid reporting of clinical studies and reviews in COPD. Special focus is given to the pathophysiological processes underlying the disease, intervention programs, patient focused education, and self management protocols.

\section{Dovepress}

This journal is indexed on PubMed Central, MedLine and CAS. The manuscript management system is completely online and includes a very quick and fair peer-review system, which is all easy to use. Visit http://www.dovepress.com/testimonials.php to read real quotes from published authors. 